\title{
Trauma management incorporating focused assessment with computed tomography in trauma (FACTT) - potential effect on survival
}

\author{
Karl-Georg Kanz ${ }^{* 1}$, April O Paul1, Rolf Lefering4, Mike V Kay'1, Uwe Kreimeier², Ulrich Linsenmaier³, Wolf Mutschler¹, \\ Stefan Huber-Wagner ${ }^{1}$ for the Trauma Registry of the German Trauma Society
}

\begin{abstract}
Background: Immediate recognition of life-threatening conditions and injuries is the key to trauma management. To date, the impact of focused assessment with computed tomography in trauma (FACTT) has not been formally assessed. We aimed to find out whether the concept of using FACTT during primary trauma survey has a negative or positive effect on survival.

Methods: In a retrospective, multicentre study, we compared our time management and probability of survival (PS) in major trauma patients who received FACTT during trauma resuscitation with the trauma registry of the German Trauma Society (DGU). FACTT is defined as whole-body computed tomography (WBCT) during primary trauma survey. We determined the probability of survival according to the Trauma and Injury Severity Score (TRISS), the Revised Injury Severity Classification score (RISC) and the standardized mortality ratio (SMR).

Results: We analysed 4.817 patients from the DGU database from 2002 until 2004, 160 (3.3\%) were from our trauma centre at the Ludwig-Maximilians-University (LMU) and 4.657 (96.7\%) from the DGU group. $73.2 \%$ were male with a mean age of 42.5 years, a mean ISS of 29.8. 96.2\% had suffered from blunt trauma. Time from admission to FAST (focused assessment with sonography for trauma)(4.3 vs. $8.7 \mathrm{~min}$ ), chest x-ray (8.1 vs. $16.0 \mathrm{~min}$ ) and whole-body CT (20.7 vs. $36.6 \mathrm{~min}$ ) was shorter at the LMU compared to the other trauma centres ( $p<0.001)$. SMR calculated by TRISS was 0.74 (CI95\% 0.40-1.08) for the LMU ( $p=0.24)$ and 0.92 (CI95\% 0.84-1.01) for the DGU group $(p=0.10)$. RISC methodology revealed a SMR of $0.69(95 \% \mathrm{Cl} 0.47-0.92)$ for the LMU $(p=0.043)$ and $1.00(95 \% \mathrm{Cl} 0.94-1.06)$ for the DGU group $(p=0.88)$.

Conclusion: Trauma management incorporating FACTT enhances a rapid response to life-threatening problems and enables a comprehensive assessment of the severity of each relevant injury. Due to its speed and accuracy, FACTT during primary trauma survey supports rapid decision-making and may increase survival.
\end{abstract}

\section{Background}

In central European trauma centres there is an increasing trend towards focused assessment with computed tomography in trauma (FACTT) [1,2]. According to the annual report 2008 of the German trauma registry, more than $44.9 \%$ reporting trauma centres utilize whole-body computed tomography (WBCT) in major trauma due to its speed and accuracy [3].

\footnotetext{
*Correspondence: karl-georg.kanz@med.uni-muenchen.de
}

${ }^{1}$ Munich University Hospital, Department of Trauma Surgery - Campus Innenstadt, Ludwig-Maximilians-University, Munich, Germany Full list of author information is available at the end of the article
In 1997 Löw was the first to consider clinical use of WBCT during initial trauma resuscitation in Mainz, Germany [4]. The first subsequent patient series was conducted by Scherer in Munich, Bavaria in 1999 [5]. Leidner in Oskarshamn, Sweden [6], Ptak in Boston, U.S.A. [7], Klöppel in Leipzig, Germany [8] and Rieger in Innsbruck, Austria [9] all followed. Additionally Mutze from Berlin, Germany, introduced a detailed whole-body CT protocol for multiple trauma patients in a in a digitized radiology department [10]. Since then several approaches to the integration of WBCT into trauma room protocols have been presented [1,2,11-14]. Recently it could be demon- 
strated that integration of WBCT into early trauma care significantly increased the probability of survival in patients with polytrauma [15].

Implementing WBCT requires a trauma room (TR) that is suitably equipped governed by an algorithm that enables effective diagnosis and treatment of injuries. According to our ATLS/ETC (Advanced Trauma Life Support /European Trauma Course) based trauma workflow the MSCT (multi slice CT) scanner (situated in the emergency department adjacent to the trauma room) is utilized immediately after the management of respiratory problems (Airway, Breathing) to detect causes of bleeding (Circulation) or intracranial pathologies (Disability).

To date, the impact of focused assessment with computed tomography in trauma (FACTT) has not been formally assessed. We felt that this was necessary especially as we apply WBCT in haemodynamically stable as well as in haemodynamically unstable trauma patients. By using data from the trauma registry of the German Trauma Society (DGU), our study investigated whether our concept of using FACTT during primary trauma survey has a negative or positive effect on survival.

\section{Methods}

Our trauma workflow [fig. 1] enhances an established comprehensive trauma protocol [1] and amalgamates ATLS/ETC standards. The algorithm is based on a scheme that is adjusted to priority and phase of trauma resuscitation. It incorporates diagnostics, evaluation and therapy and integrates the use of WBCT. According to the algorithm, respiratory $(A, B)$, circulatory $(C)$ and disability (D) problems need to be immediately identified and treated. Stethoscope (physical examination), sonography and chest $\mathrm{x}$-ray serve as basic diagnostic tools. After controlling respiratory problems and obvious external bleedings, WBCT is performed in order to detect relevant internal bleeding in the chest, abdomen/pelvis or intracranial pathology. At our institution the attending trauma surgeon supported by the anaesthesiologist and radiologist decides whether FACTT is performed or not. Exclusion criteria are patients which immediately require life-saving surgery.

In the case of traumatic cardiorespiratory arrest (TCRA), the first task is to confirm the correct positioning of the endotracheal tube and upon suspicion of tension pneumothorax, immediate chest decompression. The use of focused assessment with sonography for trauma (FAST) facilitates a more informed decision when considering resuscitative thoracotomy or the termination of resuscitation efforts.

In non-TCRA cases, respiratory assessment still takes priority. If the patient has been intubated on-scene, an esophageal intubation must be excluded and in severe respiratory distress emergency intubation performed. In the case of a tension pneumothorax, immediate chest decompression is required. Simultaneously a chest $x$-ray is obtained and sonography performed.

Contractility of the myocardium, pericardial effusion, end-diastolic volume and free fluid in the abdomen is examined by FAST combined with cardiac arrest ultrasound exam (C.A.U.S.E.) [16]. Life-threatening conditions such as cardiac tamponade or massive hypovolaemia are detected and treated at this stage. Relevant external haemorrhage is controlled by direct compression, tourniquet or if necessary by surgery. As an essential prerequisite for FACTT a large caliber intravenous line is necessary to administer contrast application.

Following transfer from the trauma room to the adjacent CT suite, the MSCT study was performed with a 4row multi-slice computed tomograph (Somatom Volume Zoom, Siemens AG, Medical Solutions, Erlangen, Germany). WBCT is defined as a scan of the head, neck, thorax, abdomen and pelvis. The head is scanned with $4 \times 1$ $\mathrm{mm}$ collimation ( $2 \mathrm{~mm}$ of slice thickness reconstruction of the bone and $4 \mathrm{~mm}$ of the parenchyma). Thorax, abdomen and pelvis are taken with $4 \times 2.5 \mathrm{~mm}$ collimation respectively and $5 \mathrm{~mm}$ slice thickness reconstruction of the parenchyma. Multiplanar reconstructions (MPR) of the cervical, thoracic and lumbar spine each with $3 \mathrm{~mm}$ slice sickness are compiled as a result. Detailed information on the LMU-WBCT-protocol are given in [17].

The first images on the CT console enable the trauma team to "look into the patient" and search for life-threatening problems and injuries that require emergency procedures and operations such as chest tube insertion, thoracotomy, laparotomy, pelvic C-clamp or CT-guided aortic balloon occlusion. After life-threatening conditions are managed or excluded, secondary survey supported by multiplanar reconstruction (MPR) is performed. Based on these findings, further management and treatment such as craniotomy or damage control surgery is determined.

We acquired our data set from the trauma registry of the German Trauma Society (DGU) which aggregates data of major trauma patients within German speaking countries and is a prospective, multicentric, standardized and anonymized data base. Every trauma patient admitted to one of the participating trauma hospitals ${ }^{* * * *}$ with an injury severity score (ISS) $\geq 16$ or ICU treatment is documented for the registry. Data anonymity is guaranteed both for the individual patient and the participating hospital. The registry comprises epidemiologic, physiologic, laboratory, diagnostic, operative, interventional and intensive care medical data as well as scoring and outcome data [18]. We used the dataset from 2002 to 2004 as the parameter "whole-body computed tomography" was first recorded in 2002 . We analyzed 4.817 patients, comparing 160 patients from our institution at the Ludwig- 
LMU trauma room workflow guided by ABCDE priorities (Airway, Breathing, Circulation, Disability, Environment)

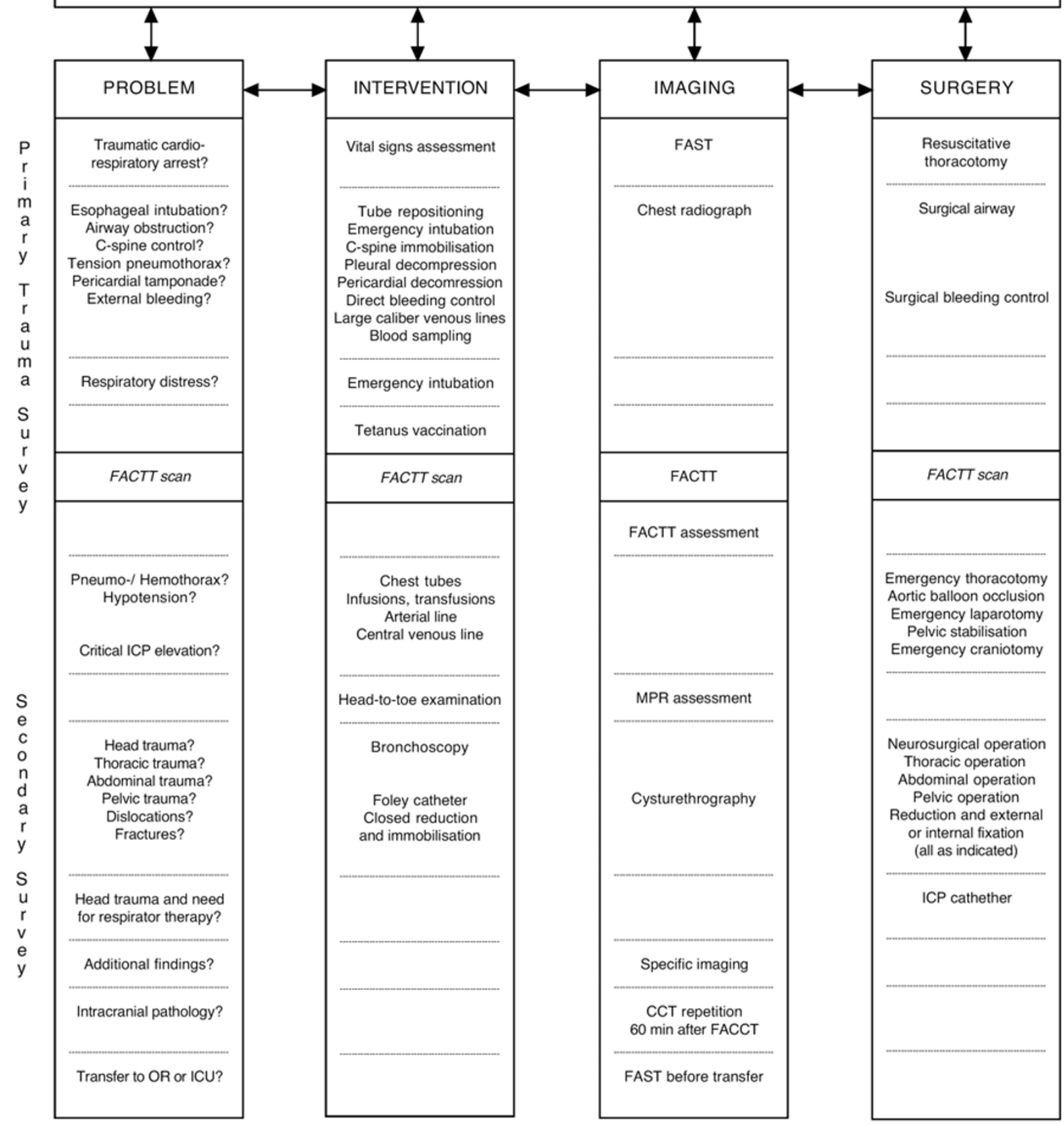

Figure 1 Ludwig-Maximilians-University (LMU) trauma room workflow. FACTT: focused assessment with computed tomography in trauma; ICP: intracranial pressure; OR: operation theatre; ICU: intensive care unit; FAST: focused assessment with sonography for trauma; MPR: multiplanar reformat/reconstruction; CCT: cranial computed tomography.

Maximilians-University Munich (LMU) to 4.657 trauma patients from other trauma centres (DGU) participating in the registry. Inclusion criteria were patients presenting with an ISS $\geq 16$, who had been admitted directly from the incident scene to the hospital and in which informa- tion about WBCT had been documented. This study has received the full approval of the ethics committee of the Ludwig-Maximilians-University of Munich, Germany.

The descriptive data analysis to compare our group against the other hospitals of the registry was performed 
using the Mann-Whitney-U test (both two sided) and $\chi^{2}$ test. The outcome analysis was carried out by calculating the Trauma and Injury Severity Score (TRISS), the Revised Injury Severity Classification score (RISC) and the standardized mortality ratio (SMR, observed/ expected mortality). Details on the TRISS and RISC score are given in [15,19-21] (TRISS) and [3,15,22] (RISC). Survival was defined as survival to discharge.

The standardized mortality ratio (SMR) is defined as a quotient of the observed to the expected mortality. If the SMR is 1 , the calculated mortality rate by score is identical with the observed mortality rate. If the SMR is less than 1 , more patients than expected survive; if the SMR is higher than 1, less patients than predicted survive. In epidemiology SMR serves as standard statistical tool.

We calculated 95\% CIs when appropriate. Significance was assessed at $\mathrm{p}<0.05$. We did the statistical analysis using SPSS (version 15.0).

To validate our trauma room concept incorporating FACTT and to analyse whether WBCT during trauma resuscitation has a negative impact on survival we performed a risk adjusted approach and compared the SMR of our university hospital (LMU) with the SMR of the other trauma centres (DGU). Furthermore we investigated the difference between LMU and other DGU centres in respect to time between admission and FAST, chest $\mathrm{x}$-ray and WBCT.

\section{Results}

4.817 patients met the inclusion criteria, they comprised of 160 (3.3\%) cases from our hospital and 4.657 (96.7\%) from the other trauma centres. All of our patients were treated according to our trauma room workflow and in 138 (86.3\%) WBCT was performed during primary trauma survey. The other 22 (13.7\%) either were dead on arrival, died before WBCT could be performed or underwent emergency surgery without CT. Table 1 gives the main characteristics of the collective.

The mean age of the whole collective $(n=4.817)$ was 42.5 years, $73.2 \%$ were male and $96.2 \%$ suffered from blunt trauma. The GCS on-scene was 10.2 points, $59.3 \%$ required endotracheal intubation, $21.4 \%$ of the patients were in shock and the mean accident to admission time was 75.3 minutes. The mean base excess on admission was -3.8 and haemoglobin concentration $11.2 \mathrm{mg} / \mathrm{dL}$. $6.4 \%$ of the patients needed an emergency operation and $77.5 \%$ of the patients were operated during stay. The mean ISS was 29.8 . The overall mortality rate was $21.9 \%$.

The results of the differences between LMU and DGU regarding time and survival are documented in table 2 .

Time from admission to FAST, chest $\mathrm{x}$-ray and WBCT was significantly shorter compared to the other trauma centres ( $\mathrm{p}<0.001)$. Especially the span of time to WBCT, which was 16 minutes shorter at our institution. TRISS could be computed for 95 (59.4\%) patients at the LMU and $2.246(48.2 \%)$ patients meeting the inclusion criteria at the other hospitals. In contrast RISC methodology could be applied for 157 (98.1\%) patients of the LMU collective and for $4.115(88.4 \%)$ at the other trauma centres. In respect to the difference between expected and observed mortality, TRISS calculation showed neither in the LMU nor in the DGU collective a significant difference (LMU p $=0.24$, DGU p $=0.10$ ). RISC calculation demonstrated a significant difference with respect to expected to observed mortality for our collective (LMU p $=0.043$, DGU $p=0.88$ ) with a SMR of 0.69 resulting in an unexpected higher survival rate.

\section{Discussion}

In a similar fashion to the acronym FAST "focused assessment with sonography in trauma" we would like to introduce the acronym FACTT "focused assessment with computed tomography in trauma" for whole-body computed tomography during primary trauma survey. We use the term "focused" not in an anatomical or topographical sense. However, the term emphasizes to focus first on the search for life-threatening injuries, second on the need for damage control surgery, and third on other injuries and surgery respectively. The prerequisite for applying FACTT is an accessible MSCT scanner, either situated in or close to the trauma room. Implementation requires a well organized interdisciplinary trauma team consisting of trauma surgeons, anaesthesiologists and radiologists.

The integration of computed tomography within clinical routine has revolutionized diagnostic radiology $[23,24]$. Since the invention of multi-slice computed tomography (MSCT), whole body computed tomography (WBCT) is possible as MSCT in comparison to singleslice CT is acquired faster. In 2001 Ptak showed that WBCT can be practiced safely in haemodynamically stable trauma patients [7]. This has been confirmed by many other studies [1,8,9,13,25-27]. Furthermore WBCT turned out to be a technique that is more time saving than applying conventional diagnostic comprising sonography, conventional radiography or non-multislice CT $[1,6-9,26,27]$ Different trauma protocols using WBCT have been compared and showed that single pass acquisition protocols entailed lower radio exposure than segmented, partially overlapping examinations [28,29]. Nevertheless, the potential risk of cancer by radiation exposure should not be neglected. Therefore an adequate use of WBCT is necessary to justify its use for ensuring fast identification of the injury pattern. The potential gain in diagnostic safety should ideally result in a higher probability of survival. That this is possible could recently be proven [15].

Our results indicate that the use of FACTT according to our trauma protocol may increase survival. RISC 
Table 1: Characteristics of 4.817 patients that met the inclusion criteria.

\begin{tabular}{|c|c|c|c|}
\hline Group & LMU & DGU & p-value \\
\hline Number & 160 & 4.657 & \\
\hline Characteristic & mean \pm SD or $\%$ & mean $\pm S D$ or $\%$ & \\
\hline \multicolumn{4}{|l|}{ Epidemiologic data } \\
\hline Age (years) & $44.6 \pm 18.3$ & $42.5 \pm 20.7$ & 0.096 \\
\hline Male gender (\%) & 75.0 & 73.2 & 0.604 \\
\hline Blunt injury (\%) & 89.4 & 96.4 & $<0.001$ \\
\hline \multicolumn{4}{|l|}{ Prehospital } \\
\hline Chest compression (\%) & 6.9 & 3.4 & 0.020 \\
\hline GCS (points) & $10.9 \pm 4.4$ & $10.2 \pm 4.8$ & 0.099 \\
\hline Chest tube insertion(\%) & 10.0 & 7.7 & 0.293 \\
\hline Intubation (\%) & 51.3 & 59.6 & 0.035 \\
\hline Shock (\%) & 23.9 & 21.4 & 0.454 \\
\hline Infusion (ml) & $1.504 \pm 866$ & $1.575 \pm 1075$ & 0.883 \\
\hline Prehospital time (min) & $61.9 \pm 51.8$ & $75.8 \pm 46.5$ & $<0.001$ \\
\hline Air lifted (\%) & 41.1 & 41.6 & 0.903 \\
\hline \multicolumn{4}{|l|}{ In-hospital } \\
\hline Chest compressions (\%) & 8.9 & 5.1 & 0.036 \\
\hline Chest tube insertion TR (\%) & 33.5 & 24.5 & 0.010 \\
\hline Shock TR SBP $\leq 90(\%)$ & 21.2 & 15.4 & 0.051 \\
\hline Base excess (mmol/L) & $-5.8 \pm 5.5$ & $-3.7 \pm 4.9$ & $<0.001$ \\
\hline Infusion (mL) & $4.950 \pm 3844$ & $2.668 \pm 2424$ & $<0.001$ \\
\hline Haemoglobin (mg/dL) & $11.3 \pm 2.9$ & $11.3 \pm 3.0$ & 0.779 \\
\hline PRBC (\%) & 40.5 & 29.6 & 0.003 \\
\hline Number of PRBC & $4.2 \pm 9.2$ & $2.6 \pm 6.6$ & 0.005 \\
\hline Thromboplastin time & $68.9 \pm 22.9$ & $74.9 \pm 23.3$ & 0.01 \\
\hline Emergency Operation (\%) & 10.6 & 6.2 & 0.025 \\
\hline Operation rate (\%) & 87.5 & 77.2 & 0.002 \\
\hline Operation per patient & $5.2 \pm 7.2$ & $2.9 \pm 3.5$ & $<0.001$ \\
\hline ICU days & $16.8 \pm 23.6$ & $12.3 \pm 14.2$ & 0.340 \\
\hline Respirator days & $14.8 \pm 22.9$ & $8.4 \pm 12.2$ & $<0.001$ \\
\hline MOF (\%) & 77.7 & 25.0 & $<0.001$ \\
\hline AIS head $\geq 3(\%)$ & 56.3 & 59.2 & 0.459 \\
\hline AIS thorax $\geq 3(\%)$ & 61.9 & 56.5 & 0.177 \\
\hline AIS abdomen $\geq 3(\%)$ & 25.6 & 23 & 0.442 \\
\hline AIS extremities $\geq 3(\%)$ & 40.6 & 36.1 & 0.239 \\
\hline ISS (points) & $32.5 \pm 16.4$ & $29.7 \pm 13.0$ & 0.296 \\
\hline NISS (points) & $40.0 \pm 17.8$ & $35.6 \pm 14.6$ & 0.006 \\
\hline Hospital days & $22.3 \pm 27.9$ & $25.9 \pm 30.1$ & 0.025 \\
\hline \multicolumn{4}{|l|}{ Outcome } \\
\hline Mortality rate (24 h) & 11.3 & 11.4 & 0.959 \\
\hline Mortality rate (overall) & 18.8 & 22.0 & 0.324 \\
\hline
\end{tabular}


Table 1: Characteristics of 4.817 patients that met the inclusion criteria. (Continued)

\begin{tabular}{llll}
\hline GOS (\%) & & 35.7 & $<0.001$ \\
5 Good recovery & 36.6 & 24.2 & $<0.001$ \\
4 Moderate disability & 22.2 & 12.7 & $<0.001$ \\
3 Severe disability & 9.8 & 3.6 & $<.001$ \\
2 Vegetative state & 11.8 & 23.8 & $<.001$ \\
1 Dead & 19.6 & & \\
\hline We analyzed 4.817 patients, comparing 160 patients from our university hospital (LMU) to 4.657 trauma patients from other trauma centres \\
(DGU) participating in the registry. SPB Systolic blood pressure; GCS Glasgow Coma Scale; ISS Injury Severity Score; NISS New Injury Severity \\
Score; ICU Intensive Care Unit; AIS Abbreviated Injury Scale; MOF Multi Organ Failure (defined as organ failure of two systems of $>2$ SOFA- \\
score points of at least 2 days duration [43]; TR Trauma room; PRBC Packed Red Blood Cells. p: X' test or Mann- Withney-U test (two sided). \\
The overall mortality rate is not exactly equal to the rate of GOS "dead" due to the fact that GOS has not been documented for all patients.
\end{tabular}

methodology confirms that in our major trauma patients the observed mortality rate was significantly lower than the expected mortality rate $(\mathrm{p}=0.043)$. On the one hand this suggests that use of FACTT combined with our trauma algorithm during primary trauma survey reduces mortality and on the other hand that our trauma room workflow leads to an efficient use of WBCT. The efficiency of our trauma protocol can be confirmed when considering the significantly shorter span of time needed to acquire sonography, chest $\mathrm{x}$-ray and computed tomography (FAST: 4.3 vs. $8.7 \mathrm{~min}$.; Chest $\mathrm{x}$-ray: 8.1 vs. 16.0 min; WBCT: 20.7 versus 36.3 min.; $\mathrm{p}<0.001$ ) compared to the other trauma centers. Reasons for this might be the possibility to perform $\mathrm{x}$-ray and sonography in the trauma room, the fact that sonography is done by the attending radiologist and not by the teamleader, the close location of the CT scanner to the trauma room and the clear aim to finish diagnostics as early as possible.

Additionally clinical parameters as the shock rate or the infusion amount as well as therapeutic measures (number of the operations) could possibly have had an influence on our results. Other potential explanations for the increased probability of survival might be the reduced time intervals from TR to ICU admission according to Ruchholtz [30]. However, these times have not been measured in our study.

We assume that trauma management incorporating FACTT provides a targeted approach during primary survey as well as during secondary survey and results in a better outcome for major trauma patients. The main advantages of FACTT are that a CT scan of all cavities enables a rapid response to life-threatening problems and that the extensive imaging enhances a comprehensive assessment of the severity of each relevant injury. Depending on the findings of FACTT, it is possible to accomplish a priority adjusted approach by deciding which injuries must be treated first. By estimating a preliminary ISS, the decision between damage control surgery and definitive care is also supported.
Furthermore FACTT often reveals unexpected or hidden diagnoses with a major therapeutic impact. Salim et al. showed that WBCT resulted in a 19\% change of treatment of the investigated 1000 patients without obvious external signs of injury [31]. Deunk et al. found out that chest or abdominal CT scans resulted in a change of treatment up to $34 \%$ in blunt trauma patients [32]. Pfeifer and Pape in their review report on a rate of $15-22 \%$ of clinically significant missed injuries in polytrauma patients [33].

Realisation of such a time sensitive trauma workflow is challenging especially for the CT technicians. Siebers et al. stated that under "front line" conditions every fifth cranial CT (CCT) and every fourth trunk CT (TCT) study was completed with a median delay of $5 \mathrm{~min}$. An independent process analysis revealed that unpreventable delays were due to system failure or patients who were unable to cooperate. Preventable delays were due to errors such as deviation from trauma room algorithm or intravenous lines that were too short [34].

It is not only the CT technicians who are challenged, all other team members need to be well managed and organized. Discroll et al. published a prospective analysis of 207 trauma patients showing that trauma teams in which staff simultaneously carry out allocated tasks have the quickest resuscitation times [35]. The recently introduced European Trauma Course (ETC) addresses this issue and focuses especially on the team approach. The team oriented ETC is evidence based, practical and flexible enough to meet regional European needs [36]

Beside a well organized trauma team, it is essential that the trauma room workflow is adapted to the specific structure of the hospital. Several studies in different settings demonstrated that implementation of a trauma room algorithm incorporating WBCT catalyses management to develop a faster, more accurate and efficient management procedure for major trauma patients to maximise the value of the WBCT $[11,12,14]$. 
Table 2: Differences between LMU and DGU trauma centres regarding time and survival.

\begin{tabular}{|c|c|c|}
\hline Group & LMU & DGU \\
\hline Number & 160 & 4.657 \\
\hline Characteristic & $\mathrm{n}$ or $\%[95 \% \mathrm{Cl}]$ or mean $\pm \mathrm{SD}$ & $\mathrm{n}$ or $\%[95 \% \mathrm{Cl}]$ or mean $\pm \mathrm{SD}$ \\
\hline \multicolumn{3}{|l|}{ Diagnostics } \\
\hline \multicolumn{3}{|l|}{ FAST } \\
\hline $\mathrm{N}$ & $125 / 160$ & $2.676 / 4.657$ \\
\hline$\%$ & 78.1 & 57.5 \\
\hline Time (min) & $4.3 \pm 3.3$ & $8.7 \pm 14.1$ \\
\hline p-value ${ }^{1}$ & \multicolumn{2}{|c|}{$<0.001$} \\
\hline \multicolumn{3}{|l|}{ Chest x-ray } \\
\hline $\mathrm{N}$ & $111 / 160$ & $2.464 / 4.657$ \\
\hline$\%$ & 69.4 & 52.9 \\
\hline Time (min) & $8.1 \pm 4.0$ & $16.0 \pm 19.9$ \\
\hline$p$-value ${ }^{1}$ & \multicolumn{2}{|c|}{$<0.001$} \\
\hline \multicolumn{3}{|l|}{ WBCT } \\
\hline $\mathrm{N}$ & $138 / 160$ & $1.223 / 4.657$ \\
\hline$\%$ & 86.3 & 26.3 \\
\hline Time (min) & $20.7 \pm 17.6$ & $36.3 \pm 28.3$ \\
\hline$p$-value ${ }^{1}$ & \multicolumn{2}{|c|}{$<0.001$} \\
\hline \multicolumn{3}{|l|}{ Outcome } \\
\hline \multicolumn{3}{|l|}{ TRISS } \\
\hline $\mathrm{N}$ & $95 / 160$ & $2.246 / 4.657$ \\
\hline$\%$ & $59.3[95 \% \mathrm{Cl} 51.8-67.0]$ & $48.2[95 \% \mathrm{Cl} 46.8-49.7]$ \\
\hline Observed mortality $n$ & $15 / 95$ & $404 / 2246$ \\
\hline Observed mortality rate $\%$ & $15.8[95 \% \mathrm{Cl} 8.5-23.1]$ & $18.0[95 \% \mathrm{Cl} 16.4-19.6]$ \\
\hline Expected mortality rate by TRISS (\%) & 21.4 & 19.5 \\
\hline SMR & $0.74[95 \% \mathrm{Cl} 0.40-1.08]$ & $0.92[95 \% \mathrm{Cl} 0.84-1.01]$ \\
\hline$p$-value ${ }^{2}$ & 0.24 & 0.10 \\
\hline \multicolumn{3}{|l|}{ RISC } \\
\hline $\mathrm{N}$ & $157(160)$ & $4.115 / 4.657$ \\
\hline$\%$ & $98.1[95 \% \mathrm{Cl} 96.0-100.0]$ & $88.4[95 \% \mathrm{Cl} 87.4-89.3]$ \\
\hline Observed mortality $n$ & $30 / 157$ & $878 / 4115$ \\
\hline Observed mortality rate $\%$ & $19.1[95 \% \mathrm{Cl} 13.0-25.3]$ & $21.3[95 \% \mathrm{Cl} 20.1-22.6]$ \\
\hline Expected mortality rate by RISC $\%$ & 27.6 & 21.4 \\
\hline SMR & $0.69[95 \% \mathrm{Cl} 0.47-0.92]$ & $0.995[95 \% \mathrm{Cl} 0.94-1.06]$ \\
\hline$p$-value ${ }^{2}$ & 0.043 & 0.88 \\
\hline
\end{tabular}

We analyzed 4.817 patients, comparing 160 patients from our university hospital (LMU) to 4.657 trauma patients from other trauma centres (DGU) participating in the registry. FAST Focused Assessment with Sonography in Trauma; WBCT whole-body Computed Tomography; TRISS Trauma Revise Injury Severity Score; RISC Revised Injury Severity Classification; SMR Standard Mortality Ratio. p: $X^{2}$ test or Mann- Withney- U test (two sided); $\mathrm{p}^{1}$ refers to the difference between LMU and the other hospitals; $\mathrm{p}^{2}$ refers to the difference between expected and observed mortality. 
Despite the advantages of FACTT, exposure to radiation remains a critical aspect and needs to be considered. For a WBCT an effective radiation dose of 10-20 mSv can be assumed, while $2 \mathrm{mSv}$ for a conventional radiography series (consisting of chest, spine, pelvis) and 5-16 mSv for a selective organ CT are reported [11,26,37,38]. The effective dose on particular organs can accumulate and therefore potentially increase individual cancer risk [39]. The dose depends on patients size and moreover on scanning parameters and on the applied trauma protocol. Richards et al. investigated different protocols, comparing spiral CT to multislice CT in order to quantify the radiation exposure effect of the protocols on lifetime cancer risk. In their institution the multislice $\mathrm{CT}$ protocols exposed the patients to less radiation than single slice CT [40].

A study design that aims to investigate the effect of early assessment with WBCT in major trauma has recently been published. This trial is called "randomized study of early assessment by CT scanning in trauma patients" (REACT). The primary objective is to prove the beneficial effects of early trauma room CT scanning on trauma patients by comparing the effects of a strategy involving early trauma room CT scanning with a standard diagnostic imaging strategy on patient outcome. This is done by the analysis of the days spent outside the hospital in the first year after the trauma. In the latter strategy, the WBCT scanner is not located in the trauma room, but elsewhere in the hospital. The secondary objectives are to document the impact of introducing trauma room WBCT-scanning on health outcome, logistics, capacity utilization, waiting times, economies of scale, substitution patterns, and investments. Furthermore the radiation dosage is calculated in both strategies based on the actual number and type of radiological examinations and related to the initial trauma performed in each patient during the first year [41].

An appropriate indication for the use of WBCT during trauma resuscitation remains controversial [42]. There have been attempts to implement either a triage rule [14] or certain parameters [11] as decision assistance. At our institution the attending trauma surgeon supported by the anaesthesiologist and radiologist decides whether FACTT is performed or not. The use of FACTT seems justified in our collective due to the fact that our patients have been severely injured with a mean ISS of 32.5. However further investigations on comprehensive indications for FACTT should be initiated.

There are several limitations to our retrospective study. TRISS calculation could be performed only in $59.4 \%$ of the Munich patients and in $48.2 \%$ of the other participating trauma centres, whereas RISC methodology was available in $98.1 \%$ of the LMU group and $88.4 \%$ of the DGU group. Thus, the data might by biased as TRISS could not be calculated for the majority of the trauma cases. But this also indicates that RISC is much easier to maintain than TRISS, which might be due to the fact that RISC does not compute the respiratory rate on-scene. The DGU trauma registry does not record the location of the MSCT scanners in respect to the trauma room or the structure of the trauma team and the management protocol. Furthermore, the extent to which other trauma centres have implemented the principles of ATLS $/$ ETC has not been reported.

\section{Conclusion}

Trauma management incorporating FACTT enables a rapid response to life-threatening problems and enhances a comprehensive assessment of the severity of each relevant injury. Furthermore FACTT might be able to reveal unexpected or hidden diagnoses with a major therapeutic impact. Implementing FACTT requires a well organized trauma team and trauma workflow adapted to the local environment. Despite the limitations of our study the data demonstrates that our trauma room workflow enables an efficient management and that the well integrated FACTT during primary trauma survey does not harm the patient, but in fact may increase survival in major trauma.

\section{Competing interests \\ The authors declare that they have no competing interests.}

\section{Authors' contributions}

KGK and SHW participated in the idea, planning, data analysis and interpretation, statistical analysis, and writing the report. AOP participated in the data analysis and interpretation and writing the report. RL participated in the planning, statistical analysis and interpretation. MVK participated in data interpretation and writing the report. UK and UL participated in data interpretation and writing the report. WM participated in data analysis and interpretation and writing the report. All authors have seen and approved the final version of the manuscript.

\section{Acknowledgements}

Trauma Registry of the German Trauma Society (Deutsche Gesellschaft für Unfallchirurgie, DGU, Sektion Notfall-, Intensivmedizin und Schwerverletztenversorgung, NIS)

A Seekamp (chairman) S Ruchholtz (chairman), R Lefering and T Paffrath (both chairmen of Trauma Registry working subgroup).

\section{***Participating hospitals (alphabetic)}

Universitätsklinik der RWTH Aachen, Zentralklinikum Augsburg, Kreiskrankenhaus Bad Hersfeld, Charité - Campus Virchow-Klinikum, Berlin, Martin-LutherKrankenhaus Berlin, Klinikum Berlin-Buch, BG-Unfallklinik Berlin-Mahrzahn, Krankenanstalten Gilead Bielefeld, BG-Klinik Bochum Bergmannsheil, Knappschaftskrankenhaus der Ruhr-Universität Bochum, Friedrich-Wilhelms-Universität Bonn, Zentralkrankenhaus Sankt-Jürgen-Straße Bremen,

Zentralkrankenhaus Bremen Ost, Klinikum Bremerhaven-Reinkenheide, Allgemeines Krankenhaus Celle, Klinikum Chemnitz, Klinikum Dessau, Klinikum Lippe-Detmold, Krankenhaus Dresden-Neustadt, Technische Universität Dresden, Krankenhaus Dresden-Friedrichstadt, Heinrich-Heine-Universität Düsseldorf, Klinikum Erfurt, Kreiskrankenhaus Eschwege, Universitätsklinikum Essen, Evang. Krankenhaus Lutherhaus Essen, BG Unfallklinik Frankfurt/Main, Universitätsklinik Frankfurt/Main, Klinikum Frankfurt/Oder, Klinikum Fürth, JohanniterKrankenhaus Geesthacht, Städtisches Klinikum Görlitz, Klinik an Eichert Göppingen, Georg-August-Universität Göttingen, Universität Graz (Österreich), Allg Unfallvericherungsanstalt Graz (Österreich), Kreiskrankenhaus Grevenbroich, 
Universitätsklinik Groningen (Niederlande), Kreiskrankenhaus Gummersbach, BG-Unfallkrankenhaus Hamburg, Kreiskrankenhaus Hameln, Medizinische Hochschule Hannover, Krankenhaus Hannover-Nordstadt, Friederikenstift Hannover, Ev. Krankenhaus Hattingen, Orthopäd. Universitätsklinik Heidelberg, St. Bernward Krankenhaus Hildesheim, Universität des Saarlandes Homburg/Saar, Waldviertel Klinikum Horn (Österreich), LKH Judenburg-Knittelfeld (Österreich), Städt. Klinikum Karlsruhe, Christian-Albrechts-Universität Kiel, Chirurgischer Lehrstuhl der Universität zu Köln, Städt. Klinikum Köln-Merheim, Allg. öff. Krankenhaus Krems/Donau (Österreich), Städt. Klinikum St. Georg Leipzig, Universität Leipzig, Ev. Krankenhaus Lengerich, Allg. öffentl. Krankenhaus Linz (Österreich), Ev. Krankenhaus Lippstadt, Universitätsklinikum Lübeck, BG Unfallklinik Ludwigshafen, St--Marien-Hospital Lünen, Krankenhaus Altstadt, Städt. Klinikum Magdeburg, Otto-von-Guericke-Universität Magdeburg, Johannes-Gutenberg-Universität Mainz, Universitätsklinikum Mannheim, Universität Marburg, Klinikum Minden, Krankenhaus Maria Hilf Mönchengladbach, Chirurgische Klinik Innenstadt der Ludwig-Maximilians-Universität München, Städt. Krankenhaus München-Harlaching, Westfälische Wilhelms-Universität Münster, BGUnfallklinik Murnau, Lukaskrankenhaus der Städt. Kliniken Neuss, Marienhospital Osnabrück, Vogtland Klinikum Plauen, Klinikum Remscheid, Klinikum Rosenheim, Sana-Krankenhaus Rügen, St. Johanns-Spital - Landeskrankenhaus Salzburg (Österr.), Diakonissenkrankenhaus Schwäbisch Hall, Kreiskrankenhaus Soltau, Johanniter-Krankenhaus der Altmark Stendal, Kreiskrankenhaus Traunstein, BG-Unfallklinik Tübingen, Bundeswehrkrankenhaus UIm, Universitätsklinik Ulm, Klinikum der Stadt Villingen-Schwenningen, Klinikum Weiden/Opfz., Asklepios Kreiskrankenhaus Weißenfels, Donauspital Wien (Österreich), Ferdinand-Sauerbruch-Klinikum Wuppertal, Julius-Maximilians-Universität Würzburg, Universitätsspital ETH Zürich (Schweiz), Rettungsstelle Zusmarshausen.

There was no funding for his particular study. The trauma registry of the German Trauma Society (Deutsche Gesellschaft für Unfallchirurgie) was partly funded by the Deutsche Forschungsgemeinschaft Ne $385 / 5$ and by a grant from Novo Nordisk A/S, Bagsvaerd, Denmark.

\section{Author Details}

${ }^{1}$ Munich University Hospital, Department of Trauma Surgery - Campus Innenstadt, Ludwig-Maximilians-University, Munich, Germany, ${ }^{2}$ Munich University Hospital, Department of Anaesthesiology - Campus Innenstadt, Ludwig-Maximilians-University, Munich, Germany, ${ }^{3}$ Munich University Hospital, Department of Clinical Radiology - Campus Innenstadt, Ludwig-MaximiliansUniversity, Munich, Germany and 4 Institute for Research in Operative Medicine, University Witten/Herdecke, Faculty of Medicine, Cologne, Germany

Received: 4 November 2009 Accepted: 10 May 2010

Published: 10 May 2010

\section{References}

1. Kanz KG, Korner M, Linsenmaier U, Kay MV, Huber-Wagner SM, Kreimeier U, Pfeifer KJ, Reiser M, Mutschler W: Priority-oriented shock trauma room management with the integration of multiple-view spiral computed tomography. Unfallchirurg 2004, 107(10):937-44.

2. Wurmb TE, Fruhwald P, Hopfner W, Keil T, Kredel M, Brederlau J, Roewer N, Kuhnigk $\mathrm{H}$ : Whole-body multislice computed tomography as the first line diagnostic tool in patients with multiple injuries: the focus on time. J Trauma 2009, 66(3):658-65.

3. Annual Report of the Trauma Registry of the German Trauma Society (DGU): Working Group on Polytrauma - German Trauma Society (DGU). 2008

4. Löw R, Duber C, Schweden F, Lehmann L, Blum J, Thelen M: Whole body spiral CT in primary diagnosis of patients with multiple trauma in emergency situations. Rofo 1997, 166(5):382-8.

5. Scherer R, Rupp P, Sebisch E, Höcherl E: Preclinical and clinical management of major trauma patients. Rettungsdienst 1999, 12(22):36-43.

6. Leidner B, Adiels M, Aspelin P, Gullstrand P, Wallen S: Standardized CT examination of the multitraumatized patient. Eur Radiol 1998, 8(9):1630-8

7. Ptak T, Rhea JT, Novelline RA: Experience with a continous, single-pass whole-body multidetector CT protocol for trauma: the three-minute multiple trauma CT scan. Emerg Radiol 2001, 8:250-256.
8. Klöppel R, Schreiter D, Dietrich J, Josten C, Kahn T: Early clinical management after polytrauma with 1 and 4 slice spiral CT. Radiologe 2002, 42(7):541-6.

9. Rieger M, Sparr H, Esterhammer R, Fink C, Bale R, Czermak B, Jaschke W: Modern $\mathrm{CT}$ diagnosis of acute thoracic and abdominal trauma. Anaesthesist 2002, 51(10):835-42.

10. Mutze S, Madeja C, Paris S, Ostermann P, Ekkernkamp A: Helical CT examination of multiple trauma patients in a digitized radiology department. Emerg Radiol 1999, 6(2):77-80.

11. Bernhard M, Becker TK, Nowe T, Mohorovicic M, Sikinger M, Brenner T, Richter GM, Radeleff B, Meeder PJ, Buchler MW, Bottiger BW, Martin E, Gries A: Introduction of a treatment algorithm can improve the early management of emergency patients in the resuscitation room. Resuscitation 2007, 73(3):362-73.

12. Hilbert P, zur Nieden K, Hofmann GO, Hoeller I, Koch R, Stuttmann R: New aspects in the emergency room management of critically injured patients: a multi-slice CT-oriented care algorithm. Injury 2007, 38(5):552-8.

13. Philipp MO, Kubin K, Hormann M, Metz VM: Radiological emergency room management with emphasis on multidetector-row CT. Eur J Radio/ 2003, 48(1):2-4.

14. Wurmb TE, Fruhwald P, Hopfner W, Roewer N, Brederlau J: Whole-body multislice computed tomography as the primary and sole diagnostic tool in patients with blunt trauma: searching for its appropriate indication. Am J Emerg Med 2007, 25(9):1057-62.

15. Huber-Wagner S, Lefering R, Qvick LM, Korner M, Kay MV, Pfeifer KJ, Reiser M, Mutschler W, Kanz KG: Effect of whole-body CT during trauma resuscitation on survival: a retrospective, multicentre study. Lancet 2009, 373(9673):1455-61.

16. Hernandez C, Shuler K, Hannan H, Sonyika C, Likourezos A, Marshall J: C.A.U.S.E.: Cardiac arrest ultra-sound exam--a better approach to managing patients in primary non-arrhythmogenic cardiac arrest. Resuscitation 2008, 76(2):198-206.

17. Korner M, Reiser M, Linsenmaier U: Imaging of trauma with multidetector computed tomography. Radiologe 2009, 49(6):510-5

18. Ruchholtz S: The Trauma Registry of the German Society of Trauma Surgery as a basis for interclinical quality management. A multicenter study of the German Society of Trauma Surgery. Unfallchirurg 2000, 103(1):30-7.

19. Champion HR, Copes WS, Sacco WJ, Lawnick MM, Keast SL, Bain LW Jr, Flanagan ME, Frey CF: The Major Trauma Outcome Study: establishing national norms for trauma care. J Trauma 1990, 30(11):1356-65.

20. Champion HR, Sacco WJ, Copes WS, Gann DS, Gennarelli TA, Flanagan ME: A revision of the Trauma Score. J Trauma 1989, 29(5):623-9.

21. Champion HR, Sacco WJ, Hunt TK: Trauma severity scoring to predict mortality. World J Surg 1983, 7(1):4-11.

22. Lefering R: Development and validation of the Revised Injury Severity Classification (RISC) score for severely injured patients. Eur J Trauma Emerg Surg 2009, 35:437-47.

23. Crawford CR, King KF: Computed tomography scanning with simultaneous patient translation. Med Phys 1990, 17(6):967-82

24. Kalender WA, Seissler W, Klotz E, Vock P: Spiral volumetric CT with singlebreath-hold technique, continuous transport, and continuous scanner rotation. Radiology 1990, 176(1):181-3.

25. Fuchs $T$, Kachelriess M, Kalender WA: Technical advances in multi-slice spiral CT. Eur J Radio/ 2000, 36(2):69-73.

26. Heyer CM, Rduch G, Kagel T, Lemburg SP, Theisinger A, Bauer TT, Muhr G, Nicolas $\mathrm{V}$ : Prospective randomized trial of a modified standard multislice CT protocol for the evaluation of multiple trauma patients. Rofo 2005, 177(2):242-9.

27. Heyer CM, Rduch GJ, Wick M, Bauer TT, Muhr G, Nicolas V: Evaluation of multiple trauma victims with 16-row multidetector $\mathrm{CT}$ (MDCT): a time analysis. Rofo 2005, 177(12):1677-82.

28. Fanucci E, Fiaschetti V, Rotili A, Floris R, Simonetti G: Whole body 16-row multislice CT in emergency room: effects of different protocols on scanning time, image quality and radiation exposure. Emerg Radiol 2007, 13(5):251-7.

29. Ptak T, Rhea JT, Novelline RA: Radiation dose is reduced with a singlepass whole-body multi-detector row CT trauma protocol compared with a conventional segmented method: initial experience. Radiology 2003, 229(3):902-5. 
30. Ruchholtz S, Lefering R, Paffrath T, Oestern HJ, Neugebauer E, Nast-Kolb D, Pape H-C, Bouillon B: Reduction in Mortality of Severely Injured Patients in Germany [Rückgang der Traumaletalität]. Dtsch Arzteb/ 2008, 105(13):225-31.

31. Salim A, Sangthong B, Martin M, Brown C, Plurad D, Demetriades D: Whole body imaging in blunt multisystem trauma patients without obvious signs of injury: results of a prospective study. Arch Surg 2006, 141(5):468-73. discussion 473-5

32. Deunk J, Dekker HM, Brink M, Vugt vR, Edwards MJ, Vugt vAB: The Value of Indicated Computed Tomography Scan of the Chest and Abdomen in addition to the Conventional Radiologic Work-up for Blut Trauma Patients. J Trauma 2007, 63:757-763.

33. Pfeifer R, Pape HC: Missed injuries in trauma patients: A literature review. Patient Saf Surg 2008, 2:20.

34. Siebers C, Stegmaier J, Kirchhoff C, Wirth S, Korner M, Kay MV, Pfeifer KJ, Kanz KG: Analysis of failure modes in multislice computed tomography during primary trauma survey. Rofo 2008, 180(8):733-9.

35. Driscoll PA, Vincent CA: Organizing an efficient trauma team. Injury 1992, 23(2):107-10.

36. Thies K, Gwinnutt C, Driscoll P, Carneiro A, Gomes E, Araujo R, Cassar MR, Davis M: The European Trauma Course--from concept to course. Resuscitation 2007, 74(1):135-41.

37. Brenner DJ, Elliston CD: Estimated radiation risks potentially associated with full-body CT screening. Radiology 2004, 232(3):735-8.

38. Wedegartner U, Lorenzen M, Nagel HD, Weber C, Adam G: Diagnostic imaging in polytrauma: comparison of radiation exposure from wholebody MSCT and conventional radiography with organ-specific CT. Rofo 2004, 176(7):1039-44

39. Tien HC, Tremblay LN, Rizoli SB, Gelberg J, Spencer F, Caldwell C, Brenneman FD: Radiation exposure from diagnostic imaging in severely injured trauma patients. J Trauma 2007, 62(1):151-6.

40. Richards PJ, Summerfield R, George J, Hamid A, Oakley P: Major trauma \& cervical clearance radiation doses \& cancer induction. Injury 2008, 39(3):347-56.

41. Saltzherr TP, Fung Kon Jin PH, Bakker FC, Ponsen KJ, Luitse JS, Scholing M, Giannakopoulos GF, Beenen LF, Henny CP, Koole GM, Reitsma HB, Dijkgraaf MG, Bossuyt PM, Goslings JC: An evaluation of a Shockroom located CT scanner: a randomized study of early assessment by CT scanning in trauma patients in the bi-located trauma center NorthWest Netherlands (REACT trial). BMC Emerg Med 2008, 8:10.

42. Snyder GE: Whole-body imaging in blunt multisystem trauma patients who were never examined. Ann Emerg Med 2008, 52(2):101-3.

43. Vincent JL, Moreno R, Takala J, Willatts S, De Mendonca A, Bruining H, Reinhart CK, Suter PM, Thijs LG: The SOFA (Sepsis-related Organ Failure Assessment) score to describe organ dysfunction/failure. On behalf of the Working Group on Sepsis-Related Problems of the European Society of Intensive Care Medicine. Intensive Care Med 1996, 22(7):707-10

doi: 10.1186/1752-2897-4-4

Cite this article as: Kanz et al., Trauma management incorporating focused assessment with computed tomography in trauma (FACTT) - potential effect on survival Journal of Trauma Management \& Outcomes 2010, 4:4

Submit your next manuscript to BioMed Centra and take full advantage of:

- Convenient online submission

- Thorough peer review

- No space constraints or color figure charges

- Immediate publication on acceptance

- Inclusion in PubMed, CAS, Scopus and Google Scholar

- Research which is freely available for redistribution

Submit your manuscript at www.biomedcentral.com/submit
C Biomed Central 